\title{
Cambios formales en la entrevista psiquiátrica. ¿Qué hay de fondo?
}

Formal changes in the psychiatric interview. What's the background?

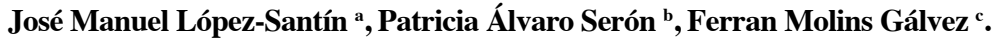 \\ ${ }^{a, b y c}$ Psiquiatra. ${ }^{a}$ INAD, CSMA Martí $i$ Julià, PSM, Barcelona, España. ${ }^{b}$ CSMA Dreta Eixample, Centre \\ Psicoterapia, Barcelona, España. ${ }^{c}$ Vuxenpsykiatrisk mottagning. Sandviken, Suecia .
}

Correspondencia: José Manuel López-Santín (25275@parcdesalutmar.cat)

Recibido: 15/04/2014; aceptado: 12/10/2014

\begin{abstract}
RESUMEN: En psiquiatría, más que en ninguna otra especialidad médica, el encuentro médicopaciente es el centro del proceso diagnóstico y terapéutico. Lo es por las propias peculiaridades del objeto de su estudio, la subjetividad. La actual perspectiva neopositivista en la epistemología de los síntomas mentales y neoliberal en la gestión de los servicios que se ocupan de lo psiquiátrico se han generalizado. De aquí se pueden derivar cambios fundamentales en diversos aspectos formales de la entrevista psiquiátrica que por fuerza afectan al contenido del acto médico. El lugar y tiempo empleados para hacer la entrevista, el entrevistador, la forma de entrevistar y la organización que provee el servicio y dispone los medios para la entrevista son algunos de ellos.

PALABRAS CLAVE: Psicopatología, subjetividad, filosofía, servicios de salud mental, administración de los servicios de salud
\end{abstract}

ABSTRACT: This article is a theoretical discussion focused on the philosophical and economical issues that affect the clinical environment. It is claimed that the manner in which psychiatry's object is regarded, as well as the management of public services, has a direct impact on the diagnostic process and therapeutical interventions. Because of the special features of psychiatry's object, which is subjectivity, the clinical encounter with the patient is at the center of the diagnostic and therapeuthic process. The current neopositivistic view on the epistemology of mental symptoms, as well as the neoliberal way of managing psychiatric services, has spread and generalized. They have brought about fundamental changes in different formal aspects of the psychiatric interview that have an effect on the medical act. Some of these formal aspects include when and where to conduct the interview, the requested interviewer characteristics, the method of interviewing and the kind of organization that provides the services as well as manages the resources.

KEY WORDS: Psychopathology, subjectivity, philosophy, mental health services, health services administration.

\section{Introducción}

La relación médico-paciente conforma uno de los pilares básicos en los que se sustenta la praxis médica. Supone el telón de fondo sobre el que se aplica la técnica médica. La recogida de la información relevante, la percepción del estado del otro de forma directa, empática y contextualizada, la importancia de la estabilidad de esa relación en lo dialogado con el paciente, la confianza establecida, etc, son todos ellos factores que contribuyen al correcto diagnóstico y al tratamiento pertinente.

En el ámbito de la psiquiatría esto se hace aún más patente, puesto que su objeto de estudio es el sujeto experienciante, y el medio fundamental para diagnosticar y tratar es el habla y la escucha en el marco del encuentro clínico. Como 
afirman Markova y Berrios (1), comprender los trastornos mentales significa aprehender su objeto en términos de su contexto histórico, social, biológico y psicológico. La relación como médico con ese objeto implica lo simbólico y lo sociohistórico. Se trata de un objeto que está en relación con él mismo, con los otros (incluido el terapeuta) y con el mundo. Como se indica repetidamente desde distintas perspectivas (2-5), el resultado de una evaluación psiquiátrica no sólo depende de lo aportado por el paciente, sino de una serie de factores que envuelven al hecho psiquiátrico, que lo modifican y condicionan. Rejón (4) ha resumido las fuentes de heterogeneidad del síntoma en cuatro:

1.- los provenientes del paciente: naturaleza, intensidad, duración y novedad de la señal biológica; y actitudes, capacidades, marco cultural y estado de conciencia del paciente.

2.- los del clínico: manera de obtener información, marco cognitivo y cultural propio y conocimientos.

3.- los del ámbito empírico: observacional-corporal reducido, observacionalcorporal expresivo y dialógico-simbólicamente preestructurado.

4.- los del modo de configurar cada ámbito empírico en síntomas: observación, comunicación e interpretación de la comunicación.

Este artículo reflexiona acerca de los cambios que está sufriendo la atención psiquiátrica en sus aspectos formales, concretamente en su método fundamental, la entrevista psiquiátrica, motivados en buena medida por los cambios sociales de las últimas décadas. Asimismo trata de volver a situar la subjetividad en el centro de la reflexión y praxis psiquiátricas, con los condicionantes y problemas que puede suponer su estudio empírico.

\section{El advenimiento del neopositivismo en psiquiatría}

Los últimos 50 años revelan un cambio de paradigma en la epistemología de los síntomas mentales. La transformación progresiva en la ontología de lo mental (la forma en que se entiende qué son los fenómenos mentales) ha dado lugar a cambios en la epistemología de lo mental (la forma en que se estudian los fenómenos mentales). En este tiempo se ha impuesto una filosofía de la ciencia que parte de los presupuestos positivistas y una concepción representacionalista de la mente. Un positivismo que se caracteriza por poseer un criterio de verdad que va de la correspondencia estricta de los enunciados con los hechos empíricos a la articulación más o menos coherente entre los diversos enunciados (6), por una presentación de los enunciados lo más formalizada posible, y por una metodología basada en la verificabilidad empírica de los enunciados primando el método inductivo. 
ORIGINALES Y REVISIONES

De ello se deriva, en el campo de la psiquiatría, la formación de un corpus orientado de una forma más instrumental que teórica, y que otorga mayor importancia a las conductas observables, y las descripciones sintéticas al margen de las distintas teorías que conforman la necesaria interpretación de las vivencias y conductas del otro. Desde su aparición en 1980, el DSM-III cumplió el criterio de cientificidad requerido por las tendencias científicas del momento con la operacionalización de criterios diagnósticos. Ese operacionalismo significaba que los conceptos serían definidos en términos de observaciones específicas o medidas (7). Se pretendía aunar las diversas conceptualizaciones de los síntomas que tienen las varias escuelas que se ocupan de la psicopatología y aumentar la fiabilidad interevaluador. Pero no se consideraron, al menos en su resultado final, las limitaciones que conllevaba. Obviar el proceso de formación de los términos psicopatológicos, derivado de una práctica clínica histórica, tiene como consecuencia la naturalización de los fenómenos mentales (4). Pero además, adoptar el método positivista comporta una tendencia a la equiparación de lo verdadero con lo útil, una instrumentalización de la relación de conocimiento, la producción de un distanciamiento de 'lo otro' a causa de esa relación instrumental y una separación de la relación de conocimiento sujeto-objeto. Y es que la verificabilidad de los conceptos se mide por su uso. Todo ello contribuye a la visión cosificada del mundo (8). Una reificación que ocurre también con los diagnósticos psiquiátricos, que en lugar de ser utilizados por su valor heurístico pasan a ser entendidos como entidades naturales (7). Además, este proceso de cambio dificulta la investigación etiológica en el campo de lo mental (9), y conduce a no pocas dificultades en el marco de la reflexión psicopatológica y en la introducción de nuevas perspectivas terapéuticas que contribuyan a ampliar y mejorar el panorama psiquiátrico. Y es que concebir el signo psiquiátrico como producto de una disfunción discreta del cerebro, asumir el valor de los síntomas prácticamente de forma única por su valencia diagnóstica más que por su papel constitutivo del proceso patológico, obviar la relacionabilidad e historicidad de un órgano abierto como es el cerebro, considerar la experiencia como simple epifenómeno de lo que ocurre realmente en el cerebro, entre otros, implica ningunear la ineludible naturaleza mediada de los síntomas psiquiátricos, la necesaria interpretación de los mismos y su indefectible comprensión a la luz de totalidades como pueden ser la historia vital, la personalidad o la estructura psíquica (10).

Finalmente, una vez cosificada la relación médico-paciente y naturalizados los fenómenos mentales se pueden operar los cambios formales en el encuentro psiquiátrico que repasamos más abajo. Es interesante recordar que la separación entre esos aspectos formales y los de contenido es una separación artificial como apuntaba Aristóteles (11). 


\section{Influencia del neoliberalismo en la praxis clínica}

Los cambios sociales del último tercio del siglo XX han influido notablemente en la estructuración de los sistemas de salud y por ende de las disposiciones implícitas en nuestro trabajo (12). La salud se convierte progresivamente en un bien de consumo, dándose con el tiempo un proceso de clientelización, con todo lo que esto implica. Juan Irigoyen (13) recorre el camino que ha transitado la praxis médica y las estructuras que la sustentan durante los últimos 40 años. Los cambios sociales tras la crisis de los 70 conllevan un proceso de reindustrialización y de reestructuración económica que implica una redefinición de las necesidades. La atención a la salud pasa a ser un bien de consumo cuyo valor se deriva de las dinámicas de los mercados, con expectativas de crecimiento indefinido. De esta forma aflora el gerencialismo como forma de gestión sanitaria. Éste se caracteriza por la evaluación (14) y una movilización práctica continua sin espacio para la reflexión, y movida por la competencia entre individuos evaluados. La evaluación mediante indicadores se convierte en obligatoria y reguladora de la praxis médica, dejando de lado la autonomía de los profesionales sanitarios. Se le da a la nueva gestión 'el papel de diseñar sistemas de información que faciliten una nueva gobernabilidad de las personas cuyas conductas sean predecibles, cuantificables y comparables. Así se construye un sujeto gestionable que responde a estímulos programados y que es portador de competencias que se renuevan'. Una vez establecidos unos estándares de calidad confeccionados desde la óptica gerencial, y habida cuenta que los profesionales autónomos y críticos se convierten en problema para el funcionamiento fluido del sistema, se dan las condiciones para reconfigurar las organizaciones sanitarias públicas de una forma más adaptada a los patrones de consumo de servicios.

La concreción de estos cambios sociales en los aspectos formales de la praxis psiquiátrica se pueden advertir mediante algunas características que enseguida se muestran.

El tiempo de las entrevistas psiquiátricas adquiere menor importancia, y progresivamente va reduciéndose. Puesto que se trata de obtención de información más que de comunicación de la experiencia, se entiende la entrevista como proceso de extracción de información. Si bien el tiempo depende del contexto de la evaluación (no es igual en urgencias, que en un dispositivo comunitario que en una unidad de hospitalización), por la propia forma de pensar los fenómenos mentales y por la propia exigencia de gestión gerencial, ese tiempo tiende a reestructurarse. De esta forma las escalas o las entrevistas estructuradas, poco aptas a lo abierto de una conversación entre dos personas y con una cada vez más tecnificación del lenguaje que dificulta la comunicación entre ambas posiciones, pasan a suplir a la entrevista abierta. Los riesgos de una aproximación estructurada han sido expuestos reciente- 
ORIGINALES Y REVISIONES

mente (15). La respuesta ofrecida se convierte más en un feedback de tipo farmacológico o una explicación causal hipotética que el reduccionismo científico más vulgar pone al alcance de la mano a los que no quieren complicarse, que en una respuesta comunicada, dialogada y no cerrada en torno a la explicación unilateral de base teórica más que experiencial. En definitiva, se trata de una separación más amplia entre los dos polos de la relación, que además implica una relación de poder más evidente. Creer que eso no afecta al proceso diagnóstico y terapéutico es vivir en una idealidad alejada de las consecuencias prácticas reales.

El espacio físico donde se da el encuentro clínico se ha transformado a la par que la sociedad que nos engloba. Vivir en una sociedad de consumo de masas, postindustrial, del trabajo inmaterial, del conocimiento (dependiendo del autor que la analice), condiciona el espacio de consulta. Éste, teniendo en cuenta las diferencies intercentros, puede ser un lugar aséptico, impersonal, al que no se otorgue ninguna importancia desde una perspectiva gerencial, llegando a ser con frecuencia minúsculo, prefabricado, con temperaturas mal reguladas y/o cambiante durante el proceso terapéutico. Otra posibilidad que cada vez más se fomenta desde el ámbito de la gestión es la de visitas virtuales, que no precisan ya ni del espacio de entrevista con el ahorro en términos económicos que ello puede suponer, pero sin valorar los aspectos clínicos que se pierden con el cambio.

El tipo de organización que se encarga de la asistencia, y que oscila entre el polo individual y el poblacional, tiende a polarizarse. Por un lado, el desarrollo de las sociedades modernas da lugar a lo que M. Foucault denomina biopolítica (16). Éste remonta al siglo XIX el desarrollo de este polo de la biopolítica centrada en el hombre-especie, es decir, el hombre en su capacidad de reproducirse, nacer, morir, durar, y en la actuación sobre las condiciones que pueden hacer variarlo. Se trata de una biopolítica que tiene a la población como sujeto y que gestiona su vida como cuerpo único mediante controles reguladores. De esta forma, la epidemiología se instituye como método de evaluación al servicio de la gestión poblacional. Es así que se instituyen métodos de evaluación que cada vez se alejan más de las necesidades personales individuales (centro del proceso asistencial terapéutico) para centrarse en las necesidades políticas, poblacionales o económicas. Un proceso que contribuye a la burocratización de las estructuras institucionales, donde es más fácil mantener el puesto de trabajo de un gestor intermedio al uso, útil para la organización burocrática del trabajo, que el de un clínico, como hacedor verdadero del trabajo.

Por otro lado, los procesos de las sociedades de la modernidad tardía orientados hacia el desarrollo de una cultura del individuo y el desenclave de las estructuras tradicionales (familia, trabajo, barrio), provocan una tendencia hacia una medicina personalizada de gestión privada que trata de dar asistencia desde una óptica comercial. Desde esta perspectiva se puede entender el enorme crecimiento 
de terapias alternativas ofertadas que, con más o menos evidencias científicas, no es esa la cuestión, aprovechan las condiciones sociales actuales. De las terapias florales hasta la farmacogenética, pasando por las múltiples medicinas orientales, todas confieren un trato más individualizado y personal que la medicina tradicional de ámbito público.

El tipo de profesional que estas organizaciones requieren para mantener tal funcionamiento cambia necesariamente. Sadler lo ha bautizado como el 'clínico genérico' (17). De ser profesiones con un alto nivel de autonomía profesional pasan a ser consideradas un obstáculo para el desarrollo de las nuevas fuerzas productivas. Se introduce así la figura de los gestores, que llevan consigo una racionalidad extraña a la racionalidad clínica de los profesionales. Pero el poder del que se les dota va contribuyendo a forjar la imagen (concretada en los nuevos profesionales contratados) de un tipo de psiquiatra válido para este nuevo modo de ejercicio médico. Ejercicio definido por 'actos médicos automatizados de baja calidad determinados principalmente por la eficiencia'. De un profesional autónomo, capaz de tomar sus propias decisiones en base a su formación y pericia clínica, formado no sólo en el programa de formación estatal requerido sino en otros ámbitos de la vida (necesarios para la mejora de la comprensión que demanda la psiquiatría), se busca un profesional heterónomo, que asuma las decisiones de gestión que se le pidan de forma acrítica y no cause problemas en el funcionamiento de un sistema altamente jerárquico y politizado y que se quiere casi automatizado. A este profesional le será precisa una constante adaptación puesto que no son las motivaciones clínicas las que dirigen los programas de atención, sino motivaciones políticas, económicas o personales curriculares del gestor institucional.

Cuanto a los métodos, repetir lo que se ha dicho más arriba. Puesto que el objetivo es el de cartografiar la intimidad del paciente se usan métodos analíticos, cuantificables y aplicables independientemente del entrevistado o entrevistador por mor de una mayor fiabilidad estadística. En otro lugar se han desarrollado algunas limitaciones de esta aproximación a expensas de los modelos dimensionales de personalidad (18). A pesar de éstas, el desarrollo de escalas no deja de crecer. El uso de entrevistas estructuradas o semiestructuradas se generaliza. Los programas informáticos de tratamiento psicológico online se están desarrollando y se presumen importantes en un futuro próximo, cosa que plantea un cambio de canal, y las consecuencias que ello pueda tener, en el acto comunicativo. Es obvia la importancia en la terapia de algunos elementos del acto médico (como son el contacto ocular, la gestualidad, el tono de voz, la proximidad, la reciprocidad, la escucha activa), como acto comunicativo que es y con el objetivo de crear una relación terapéutica adecuada para el propósito común de la mejoría clínica. Algunos estudios avalan determinadas prácticas comunicativas en psiquiatría para obtener mejores resultados clínicos (19-20). 
ORIGINALES Y REVISIONES

\section{La psicopatología manda. Retorno a la subjetividad}

Una mirada panorámica de la actualidad psiquiátrica ofrece una imagen de cambio cualitativo en el último siglo. Se ha pasado de una comunicación con el paciente a una extracción de información que el paciente posee, de la pericia clínica individual a los estándares comunes y la uniformización formativa, de una reflexión idiográfica a un análisis epidemiológico, se ha transitado por distintos marcos teóricos hasta llegar a la genética y sus promesas futuras, se ha relegado la experiencia subjetiva en pro de las conductas observadas. Este camino, si bien puede ofrecer algunos resultados, dificulta otros si no se atienden.

$\mathrm{Y}$ es que es preciso para nuestro trabajo entender que la aplicación adecuada de conceptos del lenguaje psicopatológico tiene una importante dependencia de elementos contextuales que no se encuentran en la definición de los términos psicopatológicos, pero que sí están presentes en el caso singular. Existen varios aspectos que motivan esa importancia contextual. El primero, las relaciones entre el contenido de la proferencia y las cualidades expresivas de la proferencia. Atender sólo al contenido implica el riesgo de agrupar distintos fenómenos bajo el mismo término psicopatológico. El segundo, los contextos de proferencia, el contexto comunicativo. En tercer lugar, el contexto histórico-biográfico y social del caso concreto. Y un último aspecto es el de la copresencia de otros síntomas que modulan la determinación del juicio psicopatológico final. Es por todo ello que la operacionalización del lenguaje psicopatológico tiene una utilidad limitada. Se mantienen definiciones observacionales y con un lenguaje pretendidamente ateórico para ganar fiabilidad interevaluador y combatir las distintas interpretaciones de los fenómenos que cada escuela psicopatológica hace. Pero la realidad es que el lenguaje psicopatológico está plagado de expresiones metafóricas por la misma ontología de lo mental, por su carácter inaprensible y dinámico. Así pues, en ese proceso de operacionalización se pierde información relevante para el proceso diagnóstico y terapéutico. El valor de determinados términos psicopatológicos, no es el de señalar algún tipo de entidad natural, sino el intento de capturar un fragmento de experiencia y hacerla comunicable entre una comunidad de profesionales. La correcta aplicación práctica de los criterios operacionales se da solamente bajo la condición de tener una previa familiaridad con los términos psicopatológicos. Esa familiaridad está basada en la experiencia y exige hacer un balance entre la información que incluyen las definiciones de los términos psicopatológicos y los diferentes aspectos contextuales mencionados antes.

Captar esos elementos contextuales, integrarlos en el relato discursivo del sujeto que consulta para interpretarlos desde el punto de vista psicopatológico, requiere del psiquiatra otras cualidades que no se promueven habitualmente en una sociedad tecnificada como la actual. G. Stanghellini (21) aboga por un aprendizaje humanísti- 
co de los psiquiatras, en el sentido del término gadameriano Bildung (algo así como cultivo o formación, más que educación técnica). Un conocimiento que dotaría de una capacidad de apertura al otro a través del tacto. Una cualidad que pretende que sea esencial en el psiquiatra, y que permite 'sentir lo atmosférico, sintonizar, sin meterse en la esfera del otro, evitando las relaciones instrumentales, y dejando al otro manifestar su singularidad'. Finalmente propone que esta formación es cosa de la psicopatología, puesto que en su centro está la persona que experimenta y la experiencia subjetiva. Y es que hay que tener en cuenta que el paciente es una pareja activa en el proceso diagnóstico, capaz de teorizar e interpretar sus propias demandas, y 'los síntomas deben ser conceptualizados como el resultado de la mediación entre el self encarnado vulnerable, por un lado, y la persona enferma intentando manejar y dar sentido a sus propias alteraciones y molestias, por el otro'. En este sentido revisa tres modelos contemporáneos que sitúan al paciente como sujeto activo en su proceso, en tanto es capaz de reapropiarse de su experiencia e interpretarla (22). Este proceso que el paciente realiza, no está al margen del papel que el psiquiatra juega en su relación con él. Puesto que se trata de un especialista en las alteraciones de la experiencia y el comportamiento, es una figura importante a la hora de brindar información para las interpretaciones, para guiar a través de las fases del trastorno, para co-construir las interpretaciones, etc. Y todo ello requiere de una relación de confianza, mantenida en el tiempo, de continuidad, que si no se da, muy probablemente reduzca la comunicación de las experiencias anormales y de las interpretaciones a las que el sujeto llega.

Entender la tarea de la psicopatología, como herramienta para dotar de inteligibilidad a la experiencia y conducta de un sujeto, y de la psiquiatría como práctica que se hace cargo de ésta, es lo que están haciendo algunos psiquiatras en los últimos años (23-26). En estrecha relación con la filosofía, reabren el debate en torno a lo subjetivo como ámbito propio de la psiquiatría y tratan de aclarar y orientar su estudio de forma científica, asumiendo los aprioris y limitaciones del mismo. La apuesta por métodos cualitativos (27) o la confección de nuevos instrumentos que permitan captar mejor los fenómenos mentales (28) son algunas propuestas que amplían el panorama conceptual, permiten la formulación de nuevas hipótesis y de aproximaciones prácticas más amplias, además de controlar la relevancia en el mundo real de determinadas cuestiones psicopatológicas estudiadas o de evaluar experiencias en primera persona en condiciones alejadas del laboratorio.

\section{Conclusiones}

Se observan dos aspectos históricos de la segunda mitad del siglo XX. Por un lado, el retorno al positivismo científico concretado en la psiquiatría con el de- 
ORIGINALES Y REVISIONES

sarrollo y la publicación del manual diagnóstico DSM-III y sucesivos. Y por otro, el desarrollo tardocapitalista que promueve la mercantilización de los servicios, entre ellos el de salud. Si el primero tiene gran influencia en la manera cómo se entienden los trastornos mentales, el segundo lo hace sobre las condiciones en que se los asiste. Se observa un desplazamiento del peso específico de la subjetividad en el marco teórico, derivándose una serie de consecuencias importantes (descontextualización de los síntomas, naturalización de los fenómenos mentales, reificación de los trastornos mentales), y se modifican algunas características de la entrevista psiquiátrica. Se revisan algunos aspectos formales del encuentro clínico: tiempo, espacio, profesional, organización y métodos empleados. Éstos no son ajenos a la propia tarea de la psiquiatría. Más bien son el terreno donde se da la experiencia psiquiátrica. Y por ello, marcan el desarrollo del encuentro clínico, tanto en su vertiente diagnóstica como terapéutica. Si se entiende que las manifestaciones de la enfermedad mental simplemente son epifenómenos de una patología de los sistemas cerebrales que el sujeto nos aporta y con la cual el psiquiatra identifica una entidad natural, se derivan una serie de consecuencias graves: 1) Se obvia el proceso de formación histórica de los conceptos psicopatológicos por los cuales identificamos determinados fragmentos de experiencia. De esta forma se reifican los conceptos del lenguaje psicopatológico y se tiende a naturalizar los síntomas; 2) Se olvida el sujeto como interpretador y modificador activo de sus propias experiencias. Se postula así la 'psiquiatría sin psique' que Jaspers anticipaba (29); 3) Se relativizan los aspectos formales de la entrevista, dando por sentado que el resultado de la experiencia concreta del encuentro psiquiátrico no está influido por éstos. De esta forma, se crean las condiciones de reproducción de esa misma visión.

Todo esto, deriva en una aprehensión distorsionada de los fenómenos mentales y contribuye, como viene siendo regla en las últimas décadas, a fenómenos como la comorbilidad diagnóstica sin sentido, los umbrales diagnósticos arbitrarios y las farragosas consideraciones dimensionales (29). Problemas que cada vez estorban más nuestra práctica clínica.

En definitiva, la deriva neopositivista en lo psiquiátrico y neoliberal en lo económico tiene consecuencias de gran calado en la organización formal del encuentro psiquiátrico y sus resultados. Situar la subjetividad en el centro de nuestra práctica es hacerse cargo de lo que nos ocupa, y tiene consecuencias. Será preciso adquirir las aptitudes adecuadas para ese manejo, tener el tiempo necesario para ello, tener en cuenta el espacio más adecuado para esa tarea, pensar la mejor forma de acercamiento al sujeto para una correcta evaluación. No se puede esperar una buena praxis en el trato con lo subjetivo si no se entiende que se trata precisamente con eso y se ponen las disposiciones organizacionales adecuadas. Y es que aprehender al otro, su forma de integrar y sedimentar su experiencia, requiere conocer su biografía, sus vivencias, la interpretación que hace de sí y de su experiencia, 
para poder inferir los procesos mentales que motivan las expresiones psicopatológicas y sus cambios en el tiempo. Un trabajo como el de E. Kretschmer en 'El delirio sensitivo de referencia' (30) parece imposible en la actualidad. Por eso, como clínicos, es importante defender la adecuación del tiempo, ritmo y espacio de trabajo a nuestro objeto, o demandar una formación más adecuada, o presionar para que las instituciones que se ocupan de lo mental primen lo clínico por encima de otros criterios tan en boga ahora, o exigir una valoración del profesional ajustada a su trabajo clínico. Todo ello permitirá hacer nuestra tarea con garantías y los beneficiados serán los individuos que consultan, a la sazón, cualquiera de nosotros.

\section{Bibliografia}

(1) Marková IS, Berrios GE. Epistemology of psychiatry. Psychopathology. 2012 Jan; 45(4):220-7.

(2) Vallejo Ruiloba J, Leal Cercós C. Tratado de psiquiatría. Barcelona: Ars Medica; 2010.

(3) Ramos Gorostiza P., Adán Manes J. Misunderstanding psychopathology as medical semiology: an epistemological enquiry. Psychopathology. 2011;44:205-15.

(4) Rejón Altable C. Concepción de la psicopatología como lógica : un ensayo de teoría de la psiquiatría. Madrid: Universidad Autónoma de Madrid; 2012.

(5) Berrios, G.E., Marková, I.S., Olivares JM. Retorno a los síntomas mentales: hacia una nueva metateoría. Psiquiatr biológica. 1995;2(2):51-61.

(6) Hempel C. La teoría de la verdad de los positivistas lógicos. Analysis. 1935;

(7) Hyman SE. The diagnosis of mental disorders: the problem of reification. Annu Rev Clin Psychol. 2010;6:155-79.

(8) Horkheimer M. Crítica de la razón instrumental. Madrid: Trotta; 2002.

(9) Parnas J, Bovet P. Research in psychopathology: Epistemological issues. Compr Psychiatry. 1995;36(3):167-81.

(10) Novella E. Construcción y fragmentación del sujeto psicopatológico. Arch Psiquiatr. 2010;70(1):9-24.

(11) Berrios, G.E., Marková, I., Villagran Moreno JM. ¿ Son los síntomas psiquiátricos fenómenos similares? El problema de la heterogeneidad de los síntomas. Psiquiatr Biológica. 1996;3(6):223-31.

(12) Ortiz Lobo A., Ruiz de la Mata, I. Ya es primavera en salud mental. Sobre la demanda en tiempos de mercado. Átopos. 15-22.

(13) Irigoyen J. (« el cliente siempre tiene razón»). Salud2000. 2007;(113):20-5.

(14) Irigoyen J. La metamorfosis de la evaluación. Salud2000. 2010;(129):16-9.

(15) Nordgaard J, Sass L a, Parnas J. The psychiatric interview: validity, structure, and subjectivity. Eur Arch Psychiatry Clin Neurosci. 2013 Jun;263(4):353-64.

(16) Foucault M. Historia de la sexualidad.1, La voluntad del saber. Madrid: Biblioteca Nueva; 2012 . 
ORIGINALES Y REVISIONES

(17) Sadler JZ. The instrument metaphor, hiponarrativity, and the generic clinician. In: Phillips J, editor. Philosophical perspectives on technology and psychiatry. New York: Oxford University Press; 2009. p. 23-33.

(18) López-Santín JM, Molins Galvez F, Litvan Shaw L. Trastornos de personalidad en el DSM-5: una aproximación crítica. Rev la Asoc Española Neuropsiquiatría. 2013 Sep;33(119):497510.

(19) Priebe S, Dimic S, Wildgrube C. Good communication in psychiatry-a conceptual review. Eur Psychiatry. 2011;26:403-7.

(20) Priebe S, Richardson M, Cooney M, Adedeji O, McCabe R. Does the therapeutic relationship predict outcomes of psychiatric treatment in patients with psychosis? A systematic review. Psychother Psychosom. 2011;80:70-7.

(21) Stanghellini G. The portrait of the psychiatrist as a globally minded citizen. Curr Opin Psychiatry. 2013;26:498-501.

(22) Stanghellini G, Bolton D, Fulford WKM. Person-centered psychopathology of schizophrenia: building on Karl Jaspers' understanding of patient's attitude toward his illness. Schizophr Bull. 2013;39:287-94.

(23) Stanghellini G, Ballerini M. What is it like to be a person with Schizophrenia in the social world? A first-person perspective study on Schizophrenic Dissociality--part 2: methodological issues and empirical findings. Psychopathology. 2011;44:183-92.

(24) Parnas J, Raballo A, Handest P, Jansson L, Vollmer-Larsen A, Saebye D. Self-experience in the early phases of schizophrenia: 5-year follow-up of the Copenhagen Prodromal Study. World Psychiatry. 2011;10:200-4.

(25) Møller P, Haug E, Raballo A, Parnas J, Melle I. Examination of Anomalous Self-Experience in First-Episode Psychosis: Interrater Reliability. Psychopathology. 2011. p. 386-90.

(26) Raballo A, Sæbye D, Parnas J. Looking at the schizophrenia spectrum through the prism of self-disorders: an empirical study. Schizophr Bull. 2011;37:344-51.

(27) Stanghellini G, Ballerini M. Qualitative analysis. Its use in psychopathological research. Acta Psychiatr Scand. 2008 Mar;117(3):161-3.

(28) Parnas J, Møller P, Kircher T, Thalbitzer J, Jansson L, Handest P, et al. EASE: Examination of Anomalous Self-Experience. Psychopathology. 2005;38(5):236-58.

(29) Parnas J. The RDoC program: psychiatry without psyche? World psychiatry. 2014 Feb;13(1):46-7.

(30) Kretschmer E. El Delirio sensitivo de referencia: contribución al problema de la paranoia y a la teoría psiquiátrica del carácter. Madrid: Tricastela; 2000. 